\title{
Adolescent Romantic Relationships
}

\author{
Prerna Varma ${ }^{1}$, Dr. Anurakti Mathur ${ }^{2}$
}

\section{ABSTRACT}

Adolescence is more than just a period of strife and strive. It also serves as a period of change, physical and emotional transformation- the first brush with romance. Romantic Relationships are a remarkable mystery to Indian teenagers. With Westernization of urban Indian societies, young people now tend to spend a greater deal of time being in romantic relationships, dating and thinking or talking about that perfect partner. But most parents and adults in general might dismiss as superficial. And while most adolescent relationships may last just for a few weeks or months and may not have the complexity of a committed relationship, they are certainly significant for young people and even crucial for their development. Healthy or unhealthy relationships have pertinent social and emotional effects. In this article, we summarize the theoretical framework on adolescent romantic relationships, their implications on health, adjustment, development of identity, close-relationships with peers, achievement and career and development of sexuality. It also discusses teenage pregnancy and HIV-AIDS among adolescent population.

Keywords: Adolescents, Romantic Relationship, Identity Formation, Adolescent Sexuality

Romantic relationships have been considered as a hallmark of adolescence in the West (Collins, Welsh, \& Furman, 2009). But recently, they have become popular in Indian societies as well. Globalization, Westernization and the internet boom has made these relationships more common. And while they were considered trivial or even transitory earlier, they are now regarded as significant for adolescent development (Smetana et al. 2006). The last decade has led to increase in scientific interest when it comes to adolescent romantic relationships.

Most of the adolescents and teenagers undergo the feelings of butterflies in stomach, attraction and formation of a unique bond between two people from the opposite sex. They experience such a relationship where two individuals fall in love with each other and in such a situation it is called as the romantic relationship. Basically, romantic relationships are mutually acknowledged ongoing voluntary interactions that are commonly marked by expressions of affection and perhaps current or anticipated sexual behavior (Brown et al. 1999, Furman \& Collins 2008, Manning et al. 2006). Most of the teenagers feel that they have fallen into a relationship that provides them a sense of personal satisfaction on emotional and possibly physical ground and

\footnotetext{
${ }^{1}$ Alumna, Amity Institute of Psychology and Allied Sciences, Amity University, Noida

${ }^{2}$ Professor, Amity Institute of Psychology and Allied Sciences, Amity University, Noida

(C) 2015 I P Varma, A Mathur; licensee IJIP. This is an Open Access Research distributed under the terms of the Creative Commons Attribution License (http://creativecommons.org/licenses/by/2.0), which permits unrestricted use, distribution, and reproduction in any Medium, provided the original work is properly cited.
} 
gives them a chance to grow, mature, validate their opinions, understand the concepts of love, care, trust, respect and kindness and practically apply these on the one they think they love. Most of the teenagers when start a relationship feel to be a part of those $2 \%$ of people who can develop, sustain a romantic relationship formed during teenage and consequently reach the altar.

Apart from feelings of closeness and voluntary interactions, how do these adolescent romantic relationships affect overall development of young minds? This is discussed in the next section.

\section{THE CONCEPT OF LOVE}

Millions of poems, songs and cards have been written about 'love,' but what teenagers and adolescents think about the concept of love?

Well, for some adolescents, love is larger than life, for others, it is their way of developing sexually, finding an identity, for some it is all about finding someone they want to 'cuddle with' for the rest of their lives. Some adolescents may find their 'soul-mate' at the age of 14, while a few others simply fantasize about a romantic rendezvous with the math class student they sit right next to!

Overall, love, as a concept goes beyond feelings. At its very core love may be more about the physical changes in the body than anything we might assume are psychological. The physical changes affect our psychological process and we are sprung into the euphoric feelings we associate with love. The good news is that all the complaints, bad feelings, and bodily aches may disappear when we are in love.

Love if nurtured properly leads to emotional bonding. What at earlier levels may be called as infatuation or attraction can actually become an emotional bond that requires a sense of commitment, feelings of trust, care, understanding, awareness of one's own self and the other, proper communication, sharing of ideas, thoughts, feelings and actions. Love might start as an obsession about some particular person and may culminate into the formation of a unique bond among the two that has more of psychological basis rather than physical ones. But this is not the concept of love. As the number of humans and the number of relationships increase, the concept of love may also increase. In case of a romantic relationship among two friends the concept of love may be vice-versa where the two experiences an emotional bondage far before the physical attraction, while some others who have successful long- distance relationships may not feel obsession for each other. At its very core love may be more about the physical changes in the body than anything we might assume are psychological. The physical changes affect our psychological process and we are sprung into the euphoric feelings we associate with love. The good news is that all the complaints, bad feelings, and bodily aches may disappear when we are in love.

Love if nurtured properly leads to emotional bonding. What at earlier levels may be called as infatuation or attraction can actually become an emotional bond that requires a sense of commitment, feelings of trust, care, understanding, awareness of one's own self and the other, 
proper communication, sharing of ideas, thoughts, feelings and actions. Love might start as an obsession about some particular person and may culminate into the formation of a unique bond among the two that has more of psychological basis rather than physical ones. But this is not the concept of love. As the number of humans and the number of relationships increase, the concept of love may also increase. In case of a romantic relationship among two friends the concept of love may be vice-versa where the two experiences an emotional bondage far before the physical attraction, while some others who have successful long- distance relationships may not feel obsession for each other.

The fact that the concepts of romance and love can be as unique as the number of adolescents around the world makes adolescent romantic relationships quite a curious concept for researchers. The researches on the possible effects of these relationships begin mostly after 2000, with studies beginning to understand the primary perceptions of adolescents towards their potential partners and the extent of their dating. Contemporary researchers have since then expanded their view. They are now more interested in understanding the positive and negative implications of these relationships, with great attention to cultural, racial and socio-economic diversity and elements. Others are interested to see how romantic relationships may lead to identity development, have effects on the physical changes and sexuality, impact the emotional quotient and general psychological functioning. The theoretical aspects of these relationships, their possible impact on different development factors are discussed in the following sections.

\section{REASONS BEHIND INCREASE IN ADOLESCENT DATING CULTURE IN INDIA}

Meeting socially for companionship, going beyond companionship has become quite popular in the Indian culture. Dating as a concept mayn't have been known off some two or three decades ago, but now, it is quite common, with adolescent dating culture increasing in the country. Here are some of the reasons behind the rise in dating culture.

Westernization- The increasing acceptance of Western Culture has helped urban Indians accept the concept of dating among adolescents more openly. Parents may still don't approve of adolescent romantic relationships, but they do accept it and recognize its growing occurrence. This has led to increased popularity of dating culture among adolescents.

Early Puberty- Boys and girls now experience puberty at younger ages than previous generations. In general, girls enter puberty between ages 8 and 13 and reach menarche (first menstruation) several years later, while boys enter puberty between ages 9 and 14. As they enter puberty earlier than before, boys' and girls' interest in sex increases. At the same time, they experience strong, often conflicting emotions and social pressures as they move away from childhood dependence toward more independent adulthood. Hence most of them tend to look out for romantic partners more often than before.

Peer Pressure- Most of the teenagers enter in the dating culture just for the sake of gaining acceptance among the peer group. They fear being ridiculed by their friends and their 
apprehension regarding their non-acceptance among the group if they don't follow the dating norm can actually lead to increase in number of dates.

Media Influence- Media particularly electronic and social media has these days shown love to be heaven-like. Most of the movies and television shows portray teenagers as people trying to find out their soul-mates. The concept of the "perfect one" has created indelible imprint on the minds of adolescents who too try to find for their perfect one and keep on dating till they feel that they have come across somebody who has a "connection" with their heart and soul. Adolescents now love to show off their new partner, click pictures of walking hand in hand due to the popularity of couple photos on social media.

\section{THEORETICAL PERSPECTIVES ON ADOLESCENT RELATIONSHIPS}

Biosocial Perspective- Basically, these perspectives suggest that social transformations should accompany physical transformations, a reason why adolescent romantic relationships take place in the first place. Therefore, it can be considered that social relationships and reproductive fitness or puberty occur together (Weisfeld, 1999). Human and non-human studies across the world indicate that reproductive maturation is exhibited by increasing romantic relationships and reduced physical closeness from parents. Therefore, evolutionary theory plays a role in adolescent romantic relationships (Susman, 2006)

Interpersonal Perspective- This perspective assumes that social relationships and the nature of different social processes guide individuals towards romantic relationships. Since it is considered that these relationships contribute in individual development, they begin during the time of adolescence, a period of constant change and identity formation.

Ecological Perspective- Social and cultural contexts have always encouraged humans to develop close relationships and give them special meaning or significance. This is the ecological perspective, which suggests that nature, age and other factors behind adolescent romantic relationships are shaped by current social, economic, geographical, and cultural and community conditions. That's the reason why adolescent romantic relationships, which weren't popular a few decades ago are widely popular now.

\section{THE DEVELOPMENTAL SIGNIFICANCE OF ADOLESCENT ROMANTIC RELATIONSHIPS- GOOD AND BAD}

Romantic relationships cast a crucial impact on the lives of teenagers. A healthy successful relationship can make teenagers more self-aware, confident, positive and interactive on the other hand an unsuccessful relationship can actually hinder a teenager's moral, social and intellectual development. Though most of the teenagers undergo a negative impact of the breakup of a relationship but sooner or later find ways to divert their mind and later enter into new relationships. But if teenager undergoes series of relationships, failing at each one of them can make a teenager lose self-confident, form a negative self-identity and be a crucial cause behind depression and suicide among teenagers. 
Undoubtedly it cannot be denied that teenage relationships play a primary role in helping an adolescent adapt to one's environment, social and cultural settings. For instance, A teenager who develops a strong, long lasting romantic relationship with her/his partner is normally more motivated and hence has better chances of realizing what s/he desires for. Since these adolescents have more emotional support from the member of opposite sex hence they tend to gain more knowledge about their own self and realize what they wish to gain from the society. In a successful relationship, the partner helps validate more of your positive opinion giving you a chance to adjust to the society in more creative and positive manner.

For all those teenagers who aim at establishing a romantic relationship for the sake of acceptance among a particular peer group might actually gain proper insights from their relationships. Sooner or later, teenager with such a motive realize that how and why it is not required to always follow a group norm or how not to succumb under peer pressure.

Teenagers grow physically and psychologically out of a relationship. They realize their goals and aspirations after having gone through romantic relationships.

Some teenagers might also learn important lessons in the terms of self-defense. It has researched that 1 out of every 10 teenager in a relationship is abused at some point of the time or the other. Hence, such an abusive relationship might actually make situations for a teenager worse. But at the same time it might inspire and motivate the teenager to fight for the rights of one's own self.

One of the key developmental tasks of adolescence is forming a sense of identity. Young people are in the process of refining their personal values and determining future goals. Just like relationships with family and friends, romantic relationships can facilitate the process of youth gaining a greater understanding of who they are and what they value.

Adolescent romantic relationships can also provide a training ground for youth to develop interpersonal skills. Through their dating relationships, adolescents often refine their communication and negotiation skills, develop empathy, and learn how to maintain intimate relationships. The emotional ups and downs associated with getting together and breaking up may also help youth develop important skills. While breakups may put some young people at risk for depression, they may also help youth develop emotional resiliency and coping skills needed to handle difficulties later in life (Barber \& Eccles, 2003).

As adolescents become more autonomous from their parents, their romantic relationships increasingly become a source of emotional support. One study found that, among tenth graders, only close friends provide more support than romantic partners (Furman, 2002). The role of romantic relationships as a source of support and identity formation may be especially important for sexual minority youth who are often compelled by social norms to keep their sexual orientation secret from family and friends. For sexual minority youth, their romantic partners may be the only people with whom they feel comfortable (and safe) sharing their thoughts and feelings about their sexual identity (Barber \& Eccles, 2003). 
Romantic relationships are increasingly becoming a source of emotional support for most adolescents, as they become more autonomous from their parents. Romantic relationships and their experiences, both positive and negative have an impact on emotional development. For instance, individuals who characterize their relationship experiences as positive tend to be outgoing, feel comfortable in sharing their thoughts and can be more eloquent in nature. On the other hand, those who describe their experiences in negative may have commitment issues, difficulty in staying in relationships during adulthood and may experience feelings of loneliness. Depending on the relationships, adolescents may also develop rejection sensitivity.

Basically, romantic experiences play a major role in overall individual development. Not only are they responsible for sexual development and understanding of sexual identity, they also help in developing a general sense of identity. In general, adolescents who have had positive experiences in relationships will think of themselves as attractive, while those who have had adverse romantic relationships might have little confidence in their ability to have successful relationships (Furman \& Shaffer, 2003).

Romantic relationships play a key role in sexuality. Sexual behaviors and knowledge about one's sexuality develops during this period.

Gender-related behaviors and gender-role identity is also established during this period (Hill \& Lynch, 1983). Girls may be expected to adhere to feminine stereotypes and boys may be expected to adhere masculine traits. Relationships will further intensify these behaviors. For instance, a boy who is rewarded in kisses for his beard will shave more often and act manlier, while a girl who is punished or rebuked for wearing western outfits and denims might start wearing Indian outfits like suits to comply with the demands of her boyfriend.

At the same time, significant changes take place in familial environment as well. During adolescence, the interaction between a child and parent is decreased (Larson \& Richards, 1991). While these changes don't reflect a detachment from parents, they do reflect a transformation in parent and child relationship. The process may not be a smooth one and may lead to periodic conflicts.

Romantic relationships play a major role in transformation of family relationships as well. On a basic level, adolescents may spend less time with the family members and spend more time with their romantic partners (Zimmer-Gembeck, 1999). At the same time, romantic relationships can also be a cause of conflict and tension in the family. With different expectations and parental concerns about the risks of these relationships, particularly among Indian parents, the adolescents and parents might experience conflicting situations.

During adolescence, children in India make transitions from middle school to high school, then high school to secondary school and consequently to college. Therefore, relationships during this period effect career development and scholastic achievement as well. 
Overall, early involvement in romantic relationships is linked with poorer scholastic achievement (Grinder, 1966). A negative correlation has been found between romantic involvement and sexual behavior with academic achievement. It is considered that time spent with romantic partner could be a distraction from schoolwork (but results may vary from partner to partner and their characteristics).

\section{RISKS OF ADOLESCENT ROMANTIC RELATIONSHIPS- ABUSE, TEENAGE PREGNANCY, STDS AND HIV-AIDS}

While healthy romantic relationships have many potential benefits for youth, unhealthy relationships pose risks that may have long-lasting impact. Youth are particularly vulnerable to becoming involved in relationships that include dating violence and risky sexual activity. In fact, teens report dating abuse more often than any other age group (National Center for Injury Prevention and Control, 2006).

Abuse Adolescents in dating relationships are at great risk for experiencing verbal, emotional, and physical abuse from their partners. A majority of teens (61 percent) who have been in relationships report that a partner has made them feel bad or embarrassed about themselves. More than one-fourth (27 percent) of dating teens said that they have a partner call them names or put them down. Nearly one third (30 percent) of teens who have been in relationships said that they have worried about being physically hurt by a partner and 15 percent said they have been hit, slapped, or pushed by a partner (Teenage Research Unlimited, 2006). Dating violence is not limited to heterosexual youth. One study found that sexual minority youth are more likely to have experienced dating violence than other students (Massachusetts Department of Education, 2003). Failure in relationships is also one of the largest causes of depression among Indian Youth (Saini \& Singh, 2008)

Involvement in abusive relationships can have lasting consequences for youth. Teens who have experienced physical dating abuse are more likely to be involved in intimate partner violence as adults (National Center for Injury Prevention and Control, 2006).

STDs also put teens at risk of sexually transmitted infections and pregnancy (Furman, 2002). The strongest predictor for having sexual intercourse in 7th through 12th grades is recent involvement in a romantic relationship (Bouchey\& Furman, 2003). A significant minority of teens in romantic relationships report feeling pressure to engage in sexual activity. One out of four teens report that having sex is expected if you are in a relationship and almost one-third of teen girls who had been in a relationship said that they have been pressured to have sex or engage in sexual acts when they did not want to. Additionally, nearly one-fourth of teen girls reported that they have gone further sexually in a relationship then they wanted to (Teenage Research Unlimited, 2006). Sexual activity can, of course, have long-term consequences. Almost one-third of sexually active girls report having been pregnant (Suellentrop \& Flanigan, 2006) and one out of two sexually active young people can expect to become infected with an STD by age 25 (Center for Health and Healthcare in schools, 2004). 
Teenage pregnancy is a risk that is on rise in India. Although, 18 is the marriageable age for a female, girls are married much earlier, which coupled with the rising number of adolescent relationships in the country has led to a steep rise in the Teenage pregnancy cases. But, it's not just the number of pregnant adolescents (The Times of India), but self-abortions and botched up abortions that are making doctors and psychologists concerned. With the idea of a contraception or a pill considered a taboo, most young women end up not buying them, engaging in sexual intercourse and increasing their chances of conceiving. Different data suggest that teenage pregnancy in India is as high as 62 teens out of every 1000 women in urban regions, pointing out to the growing number of pregnancies due to teenage relationships.

But that's not the only thing that concerns psychologists and healthcare personnel in general. It actually the fact that India has the third highest number of people living with HIV that is of special concern. Reports by UNAIDS suggest that 2.1 million Indians are infected with this deadly virus, with 30 percent of the population involving young adults. That's an alarming trend!

The risks need to be handled. Since adolescent romantic relationships are a growing norm in the country, it is essential that teenagers are openly taught about sex. Sex education should be made an important part of the curriculum. Students should not only be taught about sex, but even the measures that can be used for preventing STDs and pregnancy.

It is also crucial that the taboo associated with the use of contraceptives is done away with. Similar to condom dispensing machines, government must install contraceptive dispensing machines for youngsters indulging in sexual activities. Similarly, special support should be provided by community doctors and gynecologists, teaching adolescents about the correct contraception and support that they can receive from the community in case of teenage pregnancies.

But what's even more important is that parents should be supportive. Adolescents, who can openly discuss their relationships with their parents are less likely to get pregnant. It is important that parents accept the evolutionary changes in the society and discuss sexuality, abuse and other issues with their children to ensure that in case of a risk, adolescent can turn to their parents for emotional support and strength.

\section{How Psychologists can help?}

Since it is majorly psychology that can provide abundance of research on teenagers and adolescents' romantic relationships and its effects on Adolescents therefore it can be safely assumed that psychologists can help individuals during adolescence, when they come in touch with the real romance and love, concepts of dating, commitment, infatuation and long lasting intimacy, break-ups and sustenance of the romantic relationships. Psychologists can also motivate the individuals at such a crucial stage of their lives to solve their confusions, become self-aware, so that they can sustain their relationships and nurture the young love throughout their lives. 
Community and school-based programs by psychologists can succeed in helping youth develop skills for healthy relationships. Young people are very receptive to information about healthy relationships; in fact, research indicates that youth want to receive more information on relationships (Wood, Senn, Desmarais, Park, \&Verberg, 2002).

Effective programs should typically work to:

- Change attitudes toward dating violence

- Explore the negative consequences of gender stereotypes

- Help youth build conflict management, negotiation, problem solving, and anger-control skills Psychologists should also:

- Recognize the importance of peers in shaping adolescent attitudes; incorporate peer education (Wood et al., 2002; Wolfe \&Feiring, 2000)

- Be sensitive to the individual experiences of youth, considering factors such as developmental level, culture, sexual orientation, and prior dating experience (Barber \& Eccles, 2003; Centers for Disease Control, n.d.)

We cannot afford to overlook the importance of adolescence as a crucial time to teach skills for developing healthy relationships. Romantic relationships are at the center of teens' lives, providing formative experiences that can positively and negatively shape their long-term development. By teaching youth to recognize the characteristics of healthy relationships and helping them develop the communication and interpersonal skills needed to create healthy relationships, we can help ensure that they will have meaningful and fulfilling romantic relationships both in adolescence and into adulthood.

But most importantly, psychologist could perform the role of Lending ears to the teenagers who undergo problems in their romantic relationships. It is the prime concern of a psychologist to help teenagers overcome the after effects of traumatic break-ups, abuses during relationships. They only can help teenagers by understanding them and creating a favorable environment for them.

\section{DISCUSSION AND CONCLUSION}

Western thought has been long ambivalent about the role of emotion in human behavior. It has praised individuals to depict idealized deep feelings, using motifs like novels, televisions and films. And when it comes to Adolescent Romantic Relationships, the Western Society has been more open, accepting the indisputable role of romantic relationships in the emotional lives of adolescents.

India, on the other has only recently witnessed a rise in adolescent romantic relationships. This, by no means states that Romantic Relationships weren't a part of Indian culture two decades ago. What it means is the fact that these relationships weren't considered a norm or acceptable in the society two decades ago, but they are slowly getting prominent in the Indian society. Growing urbanization, changes in parenting techniques, modernization of the society are some of the 
reasons why such relationships are getting popular in Indian culture. Acceptability of these relationships is still low. And while parents have a knowledge about their kids' dating, they don't wish to acknowledge that.

In comparison to 1990s, where college students had remarkably little interaction with other sex (Verma, 1995), situations have now changed. At least 30 percent of adolescents claim to be in romantic relationships openly, while 75 percent of them answer in affirmative for relationships when asked discreetly.

It must be noted that popularity of adolescent romantic relationships has led to numerous research themes in India. Indian psychologists are now keener to observe how these relationships affect overall development of adolescents in the country.

The first, obvious effect is noticed on the 'studies' or educational performance. Comparative analysis suggest that those who have frequent romantic relationships (two or more by the age of 18 years) tend to perform lower in examinations, when compared to those that didn't. But the same studies also suggest that the effect may be temporary and that most adolescents restore their original scoring levels a few months after a relationship begins.

It's also notable that these relationships affect emotional, social and physical development of adolescents as well. In terms of physical, rise in hormones, development of sexual characteristics and acceptance of sexuality is noticed more often in adolescents who have encountered at least one romantic relationship. It is also observed that these relationships can help increase Emotional Quotient of adolescents, although it is more likely to be seen in adolescents who have sustained a relationship for at least a period of one year. Social withdrawal is often considered a negative aspect of romantic relationships during adolescence. But the researches concentrated in India have found no correlation between these factors, indicating that the levels of social activity are more or less irrespective of whether an adolescent is or isn't in a romantic relationship.

What's interesting to note here is the fact that not having a relationship can actually be distressing for the individual. During a survey of single adolescents, over 70 percent of them responded that they felt discrimination in their peer groups because they couldn't find a romantic partner. On the other hand, those with a relationship consider themselves as happier or more adjusted. Overall, romantic relationships during adolescence seem to have a lasting effect on emotional and social development. But that's just one sided opinion. Adolescents, who don't have a happy relationship tend to have emotional problems, may experience mood swings and have difficulties in adjustments.

While Romantic Relationships are now considered a norm and their effects are even vastly studied, the fact is that the perils of these relationships cannot be ignored. Indulging in sexual intercourse too early is one of those perils. Adolescents, particularly girls who indulge in sexual intercourse before the age of 18 years tend to feel misguided, cheated and depressed if left by their partner later on. At the same time, the Indian society isn't open to the concept of teenage 
pregnancies, which is now becoming a rampant problem in metropolitan cities. Apart from these obvious negative effects, researchers have noted another peril of these relationships- which comes in the form of physical, sexual or mental abuse. At least 1 in 10 females in India is abused on regular basis during adolescence by their romantic partners (Singh, 2006) and a very small population leaves or files complaint against the abuse.

Therefore, awareness programs should be conducted in schools as well as colleges to spread information about romantic relationships, the difference between infatuation and 'love' and to help students with sexual education. Parents, even if they don't accept relationships should encourage their children to discuss their attractions in school or college and be open enough to accept the concept of opposite sex attraction to ensure that adolescents can openly confide if they suffer from abuse or trauma during these relationships. Last, but not the least, students must be told that relationships and sexual attraction is common and not 'abnormal,' which can help them accept their sexuality. Further studies are needed in this field to understand the effect of this rising phenomenon, to correlate it with changes in Indian culture and to evaluate the overall effect of adolescent romantic relationships on human psyche.

\section{REFERENCES}

Albert, B., Brown, S., \& Flanigan, C. (2003). 14 and younger: The sexual behavior of young adolescents. Washington, DC: National Campaign to Prevent Teen Pregnancy.

Bouchey, H., \& Furman, W. (2003). Dating and Romantic Experiences in Adolescence . In G. Adams, \& M. Berzonsky, The Blackwell Handbook of Adolescence. Oxford, UK: Blackwell Publishers.

Collins, A., Welsh, D., \& Furman, W. (2009). Adolescent Romantic Relationships. Annual Review Psychology, 60:25.1-25 22.

Collins, W. (2003). More than myth: The developmental significance of romantic relationships during adolescence. Journal of Research on Adolescence, 13, 124.

Connolly, J., Craig, W., Goldberg, A., \& Pepler, D. (2004). Mixed Gender groups, dating and romantic relationships in early adolescence. Journal of Research on Adolescence, 14(2) 185-207.

Erickson, E. (1968). Identity, Youth and Crises. New York: Norton.

Furman, W. (2002). The emerging field of adolescent romantic relationships. Current Directions in Psychological Science, 11, 177-180.

Furman, W., \& Wehner, E. (1997). Adolescent Romantic Relationships: a developmental perspective. In S. Shulman, \& C. WA, Romantic Relationships in Adolescence: Developmental Perspectives (pp. 21-36). San Francisco: Jossey-Bass.

Halpern, C. (2003). Biological influences on adolescence romance and sexual behavior. In P. Florsheim, Adolescent Romantic Relations and Sexual Behavior: Theory, Research and Practical Implications (pp. 57-84). Mahwah: Erlbaum.

Larson, R., Csikszentmihalyi, M., \& Graef, R. (1980). Mood Variabolity and Psychosocial Adjustment of Adolescents. Journal of Youth and Adolescence, 9,469-490. 
Saini, S., \& Singh, J. (2008). Gender Differences in Relational Aggression and Psychosocial Problems in Romantic Relationships among Youth. Journal of the Indian Academy of Applied Psychology, 279-286.

Simon, V., Aikins, J., \& Prinstein, M. (2008). RomanticPartner Selection and socialization during early adolescence. Child Development.

Suellentrop, K., \& Flanigan, C. (2006). Pregnancy among sexually experienced teens, 2002. Washington, DC: The National Campaign to Prevent Teen Pregnancy.

Welsh, D., Grello, C., \& M.S, H. (2003). When love hurts: depression and adolescent romantic relationships. In P. Florsheim, Adolescent Romantic Relations and Sexual Behavior (pp. 185-211). Mahwah: Erlbaum.

Wood, E., Senn, C., Desmarais, S., Park, L., \& Verberg, N. (2002). Sources of information about dating and their perceived influence on adolescents. Journal of Adolescent Research, 17(4) 401-417.

Zimmer-Gembeck, M. (1999). Stability, Change and Individual Differences in Involvment with friends and romantic partners among adolescent females. Journal of Youth ADolescent, 28 419-38.

Zimmer-Gembeck, M., Siebenbruner, J., \& Collins, W. (2004). A prospective study of intraindividual and peer influences on adolescents' heterosexual romantic and sexual behavior. Archives Sexual Behavior, 33 381-94. 